\title{
Central Tumor Necrosis of a Large Meningioma Following Acute Anemia Caused by Hysterectomy -Case Report-
}

\author{
Tadashige KANO, Masahito KOBAYASHI, \\ Kazunari YOSHIDA, and Takeshi KAWASE \\ Department of Neurosurgery, Keio University School of Medicine, Tokyo
}

\begin{abstract}
A 55-year-old woman, with a one-year history of headache, gait disturbance, and slight aphasia, was transferred to our hospital after head injury. Magnetic resonance (MR) imaging and computed tomography revealed a large meningioma with peripheral edema. However, priority was given to previously planned gynecological surgery for uterine cancer. She developed severe anemia after the surgery, followed by consciousness disturbance. MR imaging revealed spontaneous tumor necrosis. The meningioma was totally removed. Her consciousness improved, and she was discharged with no neurological deficit. Patients with large meningioma may suffer deterioration of symptoms due to central tumor necrosis triggered by acute anemia.
\end{abstract}

Key words: meningioma, central necrosis, MIB-1

\section{Introduction}

Meningioma is the most common type of extra-axial brain tumor, accounting for $13-26 \%$ of primary intracranial neoplasms. ${ }^{8)}$ Computed tomography (CT) and magnetic resonance (MR) imaging show meningiomas typically as sharply demarcated, extra-axial mass lesions with dense, homogeneous contrast enhancement. ${ }^{4)}$ However, approximately $10-15 \%$ of benign meningiomas have atypical neuroradiological patterns such as rim-like enhancement or cyst formation, which may indicate central necrosis. ${ }^{1,2,17)}$ The mechanism of central necrosis has not been clarified in detail, and only a few reports have documented progressive central necrosis with serial neuroradiological images. ${ }^{18)}$

Here we describe a case of meningioma with acute deterioration due to central necrosis triggered by acute anemia, with serial neuroradiological images.

\section{Case Report}

A 55-year-old woman was transferred to our hospital after head injury. She had a 1-year history of headache, gait disturbance, and slight aphasia. Her Glasgow Coma Scale score was 14 (E4V4M6) with slight disorientation, and she had urinary incontinence. Head CT revealed a huge tumor in the left frontal lobe. She was admitted on the same day. MR imaging with contrast medium showed a well-demarcated tumor with almost homogeneous enhancement and peritumoral edema (Fig. 1). She had a severe allergy to iodine contrast, so angiography was not performed.

Received September 18, 2008; Accepted April 2, 2009
Before the present admission, surgical intervention had already been planned under a diagnosis of uterine carcinoma and myoma uteri by the Gynecological Department of our hospital. Priority was given to the gynecological surgery considering the possibility of malignancy and rehabilitation after surgery for brain tumor. Abdominovaginal hysterectomy was performed 2 weeks after admission. She developed severe anemia with hemoglobin $6.5 \mathrm{~g} / \mathrm{dl}$ because of intraoperative hemorrhage. Two days later, she developed consciousness disturbance with Glasgow Coma Scale score of 10 (E2V3M5) and right hemiparesis. CT showed a low density area in the tumor and enlargement of the peripheral edema. MR imaging revealed central tumor necrosis that had occurred spontaneously after the gynecological surgery (Fig. 2). She was treated initially only with iron preparation and her severe anemia persisted for one week, until blood transfusion. However, normal blood pressure was maintained during the perioperative period.

Bilateral frontal craniotomy was performed 2 weeks after the hysterectomy. The tumor was soft and grayish, and easy to suction with an ultrasonic device. The margin between the tumor and normal brain was clearly visible. The falx with the tumor attachment was also removed. Yellowish necrotic tissue was observed within the tumor. Total tumor removal with Simpson grade 1 was achieved.

Histological examination showed meningioma with marked degeneration, necrosis, and occasional atypical nuclei, but without atypical nuclear mitosis (Fig. 3A, B). MIB-1 index was low in most areas, but exceeded $10 \%$ in some areas (Fig. 3C, D). After the operation she became alert, and the hemiparesis was improved. She was discharged 1 month after the second operation without neu- 

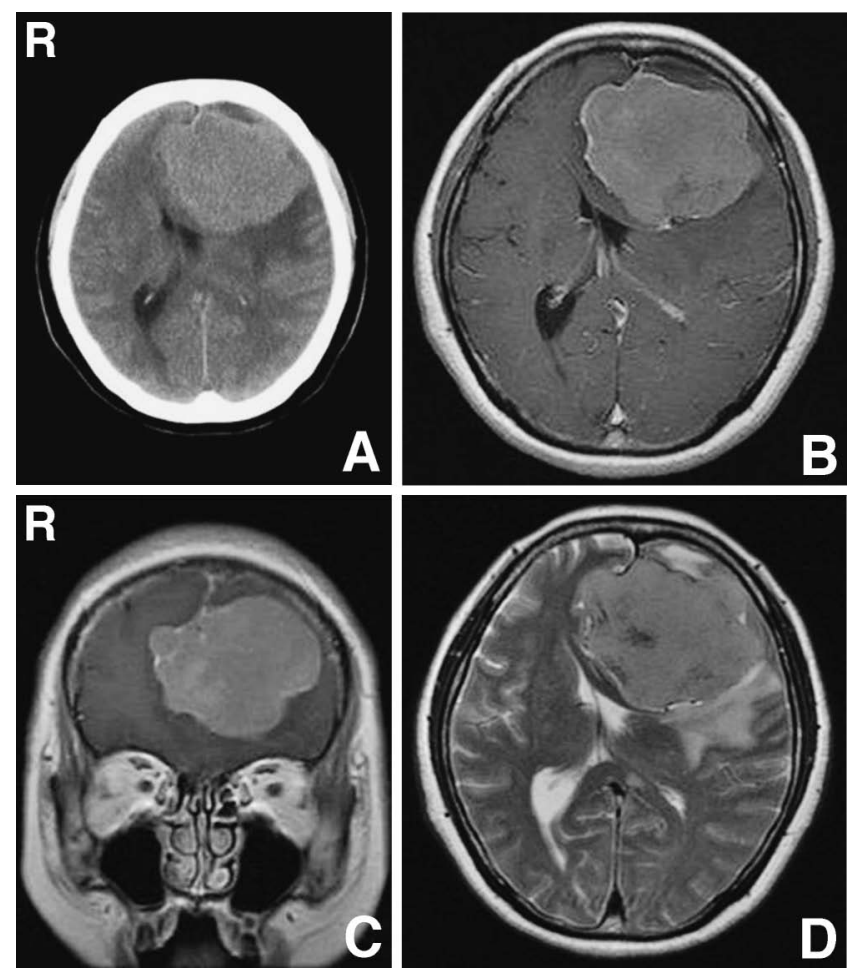

Fig. 1 A: Initial computed tomography scan showing a homogeneous isodense mass in the left frontal lobe. B, C: Axial (B) and coronal (C) $T_{1}$-weighted magnetic resonance images with gadoliniumdiethylenetriaminepenta-acetic acid showing a well-demarcated mass lesion, which is enhanced almost homogeneously but with partial strong enhancement by contrast medium. $D$ : Axial $T_{2^{-}}$ weighted magnetic resonance image showing mild peritumoral edema. Partial low intensity area is observed in the tumor.
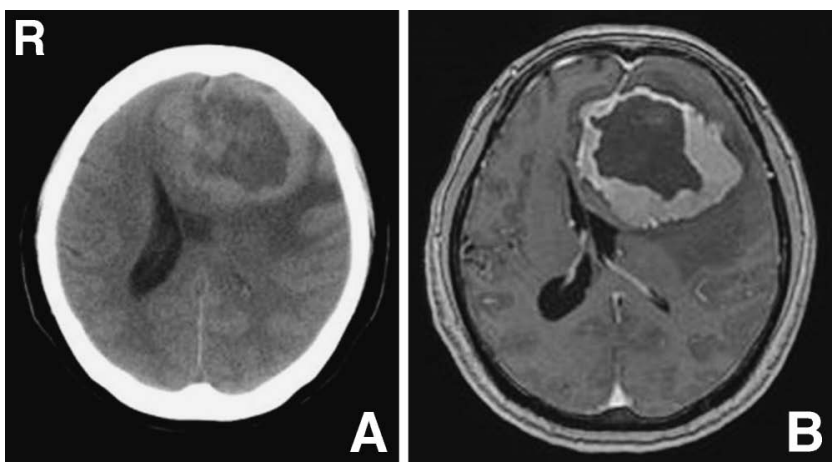

Fig. 2 A: Computed tomography scan after the hysterectomy showing a low density lesion within the isodense tumor and increased peritumoral edema. B: Axial $\mathrm{T}_{1}$-weighted magnetic resonance image with gadolinium-diethylenetriaminepenta-acetic acid showing a hypointense lesion without enhancement in the tumor, with increased midline shift.

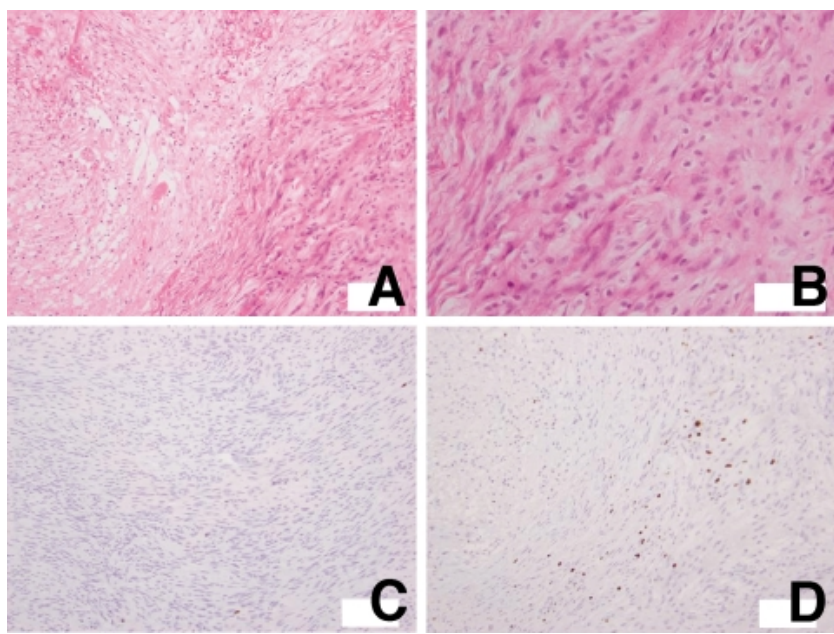

Fig. 3 A, B: Photomicrographs of the surgical specimen showing meningioma with marked degeneration, necrosis, and occasional atypical nuclei. Hematoxylin-eosin stain, original magnification A: $\times 200$; B: $\times 400$. C, D: Photomicrographs showing low MIB-1 indices in most areas (C), and perinecrotic accentuation of MIB-1 activity to about $10 \%$ (D: the same area with A). MIB-1 stain, original magnification $\times 200$.

rological deficits.

\section{Discussion}

Central necrosis is defined as massive breakdown of a large tumor with cyst formation, and should be distinguished from focal necrosis, which is suggested to be a risk factor of recurrence. ${ }^{2,3)}$ The most common mechanism of central necrosis in meningioma is suggested to be ischemia caused by sudden occlusion of tumor feeding arteries. ${ }^{11)}$ However, present histological specimens show no obstructive vessel in the viable tumor. Islands of surviving meningioma cells adjacent to the remaining patent vessels surrounded by extensive necrosis is a histological characteristic called the oasis phenomenon, and strongly suggests ischemic necrosis. ${ }^{10,17)}$ The oasis phenomenon was not clearly observed in our case, but necrosis was remarkable in the center of the tumor. The surface of the tumor was not necrotic, presumably due to adequate blood supply from the superficial feeding arteries. Therefore, we suggest that the postoperative severe anemia caused ischemic change in the tumor, resulting in the central necrosis. A previous case of meningioma showed central necrosis after cardiopulmonary arrest, indicating a strong relationship between central necrosis and ischemia. ${ }^{15)}$ Interestingly, this patient did not develop any ischemic changes in her brain, as in our patient, indicating that meningioma cells may be more vulnerable to ischemic change than normal brain tissue.

In our case, the MIB-1 index was relatively high at about $10 \%$ in some areas of the tumor. Meningiomas show occasionally tumor necrosis after embolization. High MIB-1 
proliferative indices of up to $7.1-18.5 \%$ were found in 4 of 15 cases of benign meningioma after embolization. ${ }^{14)} \mathrm{High}$ MIB-1 indices were also found in 9 embolized meningiomas with necrosis compared with 13 cases without necrosis. ${ }^{12)}$ Therefore, high MIB-1 indices in post-embolized meningiomas may indicate a temporary reactive response to necrosis, and not high grade (atypical and malignant) meningioma. ${ }^{12-14)}$ In our case, most tumor areas had low MIB-1 indices and no mitotic figures. Consequently, the histological diagnosis was benign meningioma with central necrosis, not atypical meningioma.

In cases of intravascular embolization of meningiomas, tumor necrosis occurs 24 hours after feeding artery occlusion and tumor swelling is observed a few days later. ${ }^{6,7,16)}$ Similarly, our patient showed subacute deterioration following the acute progress of anemia due to gynecological surgery. Several cases have shown acute or subacute symptomatic deterioration associated with central necrosis. ${ }^{5,9,11,18)}$ Since our patient already had a large tumor and massive edema, progression of additional perifocal edema and tumor swelling could have caused the worsening in neurological symptoms after central necrosis.

The present case provides indications for better clinical management for large meningioma, and emphasizes the possibility of acute deterioration due to tumor ischemia and necrosis triggered by other systemic disease.

\section{References}

1) Buetow MP, Buetow PC, Smirniotopoulos JG: Typical, atypical, and misleading features in meningioma. Radiographics 11: 1087-1106, 1991

2) Crompton MR, Gautier-Smith PC: The prediction of recurrence in meningiomas. J Neurol Neurosurg Psychiatry 33: 80-87, 1970

3) de la Monte SM, Flickinger J, Linggood RM: Histopathologic features predicting recurrence of meningiomas following subtotal resection. Am J Surg Pathol 10: 836-843, 1986

4) Harting I, Hartmann M, Bonsanto MM, Sommer C, Sartor K: Characterization of necrotic meningioma using diffusion MRI, perfusion MRI, and MR spectroscopy: case report and review of the literature. Neuroradiology 46: 189-193, 2004

5) Hayashi $K$, Takahata H, Nakamura M, Iwasaki K: [A case of atypical meningioma associated with acute deterioration and cerebral herniation]. No Shinkei Geka 31: 1309-1313, 2003 (Jpn, with Eng abstract)
6) Jungling FD, Wakhloo AK, Hennig J: In vivo proton spectroscopy of meningioma after preoperative embolization. Magn Reson Med 30: 155-160, 1993

7) Kai Y, Hamada J, Morioka M, Yano S, Todaka T, Ushio Y: Appropriate interval between embolization and surgery in patients with meningioma. AJNR Am J Neuroradiol 23: 139-142, 2002

8) Kleihues P, Cavenee WK: Pathology and Genetics of Tumors of the Nervous System. Lyon, IARC, 2000, pp 176-184

9) McDowell D, Harper CG: Central tumour necrosis: a cause for the acute presentation of benign meningiomas. Aust $\mathrm{N} \mathrm{Z}$ J Surg 61: 553-556, 1991

10) Miyata $I$, Tsuno $K$, Masaoka $T$, Nishiura $T$, Harada $Y$, Ishimitsu $\mathrm{H}$ : [Atypical computed tomographic features of meningioma: a case report]. No Shinkei Geka 17: 297-300, 1989 (Jpn, with Eng abstract)

11) Murai N, Kaneko T: [Three cases of fibrous meningioma with prominent central necrosis]. No Shinkei Geka Journal 16: 135-140, 2007 (Jpn, with Eng abstract)

12) Ng HK, Poon WS, Goh K, Chan MS: Histopathology of postembolized meningiomas. Am J Surg Pathol 20: 1224-1230, 1996

13) Patsouris E, Laas R, Hagel C, Stavrou D: Increased proliferative activity due to necroses induced by pre-operative embolization in benign meningiomas. J Neurooncol 40: 257-264, 1998

14) Paulus W, Meixensberger J, Hofmann E, Roggendorf W: Effect of embolisation of meningioma on Ki-67 proliferation index. J Clin Pathol 46: 876-877, 1993

15) Pullicino P, Wilbur DC, Levy RJ, Eskin TA, Kido DK: Infarction in a meningioma after cardiac arrest. Computed tomographic and pathologic findings. Arch Neurol 40: 456-457, 1983

16) Rosen CL, Ammerman JM, Sekhar LN, Bank WO: Outcome analysis of preoperative embolization in cranial base surgery. Acta Neurochir (Wien) 144: 1157-1164, 2002

17) Russell EJ, George AE, Kricheff, II, Budzilovich G: Atypical computed tomography features of intracranial meningioma: radiological-pathological correlation in a series of 131 consecutive cases. Radiology 135: 673-682, 1980

18) Terasaki KK, Zee CS: Evolution of central necrosis in a meningioma: CT and MR features. J Comput Assist Tomogr 14: 464-466, 1990

Address reprint requests to: Tadashige Kano, M.D., Department of Neurosurgery, Keio University School of Medicine, 35 Shinano-machi, Shinjuku-ku, Tokyo 160-8582, Japan. e-mail: tadashige@z6.keio.jp 